\title{
Meetings and Conferences
}

Visc Med 2018;34:162-164

31.05.-02.06.2018

Leipzig

Germany

.................

06.06.-08.06.2018

Vienna

Austria

06.06.-08.06.2018

Berlin

Germany

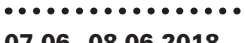

Dortmund

Germany

08.06.-09.06.2018

Ludwigshafen

Germany

09.06 .2018

Wiesbaden

Germany

................

13.06.-15.06.2018

Cologne

Germany

…...0.0.0.0.0.

13.06.-16.06.2018

Berlin

Germany

•...............

14.06.-15.06.2018

Munich

Germany

....................

14.06.-16.06.2018

Salzburg

Austria

................

15.06.-16.06.2018

Hamburg

Germany

16.06.2018

Munich

Germany

................

20.06.-23.06.2018

Cologne

Germany

.................

20.06.-23.06.2018

Barcelona

Spain

-................

21.06.-23.06.2018

Kassel

Germany

•................

29.06.-30.06.2018

Hanover

Germany
1. Gemeinsame Jahrestagung der Mitteldeutschen Chirurgenvereinigung und der Mitteldeutschen Gesellschaft für Gastroenterologie

59. Österreichischer Chirurgenkongress - Personalisierte Chirurgie

Hauptstadtkongress 2018 Medizin und Gesundheit

Viszeralmedizin NRW 2018

6. Ludwigshafener GastroTage

14. Interdisziplinäres Update - GI-Oncology 2018

50. Gemeinsame Jahrestagung der DGIIN \& ÖGIAIN

50th European Pancreas Club Meeting

46. Jahrestagung der Gesellschaft für Gastroenterologie in Bayern

51. Jahrestagung und 29. Fortbildungskurs der Österreichischen

Gesellschaft für Gastroenterologie und Hepatologie

Anorektaler Endosonographiekurs

Zertifikat Hepatologie - Update 2018

KIT 2018 - Kongress für Infektionskrankheiten und Tropenmedizin

ESMO World Congress on Gastrointestinal Cancer 2018

Ernährung 2018 - Ernährung ist Therapie und Prävention

15. HepNet Symposium
Information: www.mgfg.de

Information: www.chirurgenkongress.at

Information: www.hauptstadtkongress.de

Information: www.viszeralmedizin-nrw.de

Information: www.gastrotagelu.de

Information: www.gi-oncology.de

Information: www.2018.dgiin.de

Information: www.epc2018.com

Information: www.gfgb.org

Information: www.oeggh.at/veranstaltungen

Information: www.klinik-bergedorf.de/events/ anorektaler-endosonographiekurs

Information: www.gfgb.org

Information: www.kit2018.de

Information: www.esmo.org/Conferences/

World-GI-2018-Gastrointestinal-Cancer

Information: www.ernaehrung2018.de

Information:

www.deutsche-leberstiftung.de/symposium

\section{KARGER}


South Korea

•..............

18.07.-20.07.2018

Garmisch-

Partenkirchen

Germany

…...........

30.08.-31.08.2018

Cologne

Germany

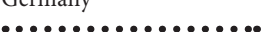

07.09.-09.09.2018

Vienna

Austria

•.............

12.09.-15.09.2018

Munich

Germany

•.................

16.09.-19.09.2018

Vienna

Austria

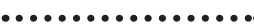

27.09.-29.09.2018

Leipzig

Germany

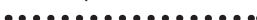

28.09.-02.10.2018

Vienna

Austria

...............

04.10.-05.10.2018

Singen

Germany

................

05.10.-10.10.2018

Philadelphia, PA

USA

.................

10.10.-12.10.2018

Budapest

Hungary

•..............

20.10.-24.10.2018

Vienna

Austria

•..............

01.11.-03.11.2018

Hamburg

Germany

•................

08.11.-10.11.2018

Mannheim

Germany

•.... . . . . . . . .

09.11.-10.11.2018

Wiesbaden

Germany

-..............

09.11.-13.11.2018

San Francisco, CA

USA

.................

15.11.-17.11.2018

Berlin

Germany

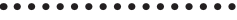

15.11.-17.11.2018

Mannheim

Germany

000000000000000

30.11.-01.12.2018

Augsburg

Germany 9th Asia-Pacific Primary Liver Cancer Expert Meeting (APPLE 2018)

95. Jahrestagung der Vereinigung Bayerischer Chirurgen (VBC) Ganzheitliche Chirurgie

GastroTage 2018 am Rhein

ECCO 2018 - European Cancer Summit

Viszeralmedizin 2018

16th World Congress of the International Society for Diseases of the

Esophagus

13. Jahrestagung der Deutschen Gesellschaft Interdisziplinäre Notfallund Akutmedizin (DGINA)

Jahrestagung der Deutschen, Österreichischen und Schweizerischen Gesellschaften für Hämatologie und Medizinische Onkologie

Der Mittelrheiner 2018 - 106. Jahrestagung der Vereinigung Mittelrheinischer Chirurgen

Annual Meeting American College of Gastroenterology

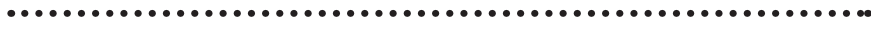

38th ESSO Congress of the European Society of Surgical Oncology

26th United European Gastroenterology (UEG) Week 2018

Endo Club Nord 2018

21. DGVS-Seminar Onkologische Gastroenterologie - Modul 2

12. Diabetes Herbsttagung der Deutschen Diabetes Gesellschaft (DAG) und 34. Jahrestagung der Deutschen Adipositas-Gesellschaft (DAG)

The Liver Meeting 2018

15. AlO-Herbstkongress - Update Medical Oncology

19. DGVS-Seminar Chronisch entzündliche Darmerkrankungen - Modul 2

endo-update 2018
Information: www.applecongress.org

Information: www.vbc2018.de

Information: www.gastrotage-am-rhein.de

Information: www.ecco-org.eu/ECCO2018Summit

Information: www.viszeralmedizin.com

Information: www.isde2018.org

Information: www.dgina-kongress.de

Information:

www.haematologie-onkologie-2018.com

Information: www.der-mittelrheiner.de

Information: acgmeetings.gi.org

Information: www.ecco-org.eu/Events/ESSO38

Information: www.ueg.eu/week

Information: www.endoclubnord.de

Information:

www.dgvs.de/fortbildung-aktuell/dgvs-veranstaltungen

Information: www.herbsttagung-ddg.de

Information: www.aasld.org/

events-professional-development/liver-meeting

Information: www.aio-herbstkongress.de

Information:

www.dgvs.de/fortbildung-aktuell/dgvs-veranstaltungen

Information: www.endoupdate.de 
$05.12-08.12 .2018$

Berlin

Germany

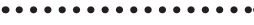

05.12.-08.12.2018

Bangkok

Thailand

..................

07.12.-08.12.2018

Amsterdam

The Netherlands

07. . . . . . . . .

07.02.-09.02.2019

Düsseldorf

Germany

06.03.-09.03.2019

Berlin

Germany

.................

02.06.-05.06.2019

Amsterdam

The Netherlands

..................

21.09.-24.09.2019

Istanbul

Turkey
17. DGVS-Seminar Hepatologie - Modul 1

Information:

www.dgvs.de/fortbildung-aktuell/dgvs-veranstaltungen

Gastro 2018: WGO-GAT International Conference

International Viral Hepatitis Elimination Meeting (IVHEM)

21st Düsseldorf International Endoscopy Symposium

17. DGVS-Seminar Hepatologie - Modul 20

13th Congress of the European-African Hepato-Pancreato-Biliary Association (E-AHPBA)

World Congress of Gastroenterology 2019
Information: www.gastro2018bangkok.com

Information: www.virology-education.com/event/

Information: www.endo-duesseldorf.com

\section{Information:}

www.dgvs.de/fortbildung-aktuell/dgvs-veranstaltungen

Information: www.e-ahpba2019.com

Information: www.worldgastroenterology.org/

meetings-and-events/meetings-and-events-calendar upcoming/5th-ivhem-2018/ 